\title{
Spiroergometrische Referenzwerte für die sozial- medizinische Leistungsbeurteilung bei Erwachsenen im Alter über 60 Jahre
}

\author{
Spiroergometric Reference Values for the Sociomedical Assessment \\ of Performance in Adults Aged over 60 Years
}

Autoren

Institut
M. Funk, J. Schneider

Institut und Poliklinik für Arbeits- und Sozialmedizin der Justus-Liebig Universität Gießen (komm. Leiter: Prof. Dr. med. Joachim Schneider) $\begin{array}{lll}\text { eingereicht } & \text { 6.2. } 2012\end{array}$ akzeptiert nach Revision 16. 3. 2012

\section{Bibliografie}

Dol http://dx.doi.org/ 10.1055/s-0032-1309362 Online-Publikation: 10.5.2012 Pneumologie 2012; 66: 329-337 (c) Georg Thieme Verlag KG Stuttgart · New York ISSN 0934-8387

\section{Korrespondenzadresse}

Prof. Dr. med.

Joachim Schneider

Institut und Poliklinik für Arbeits- und Sozialmedizin Universitätsklinikum Gießen Aulweg 129/ III 35385 Gießen Joachim.Schneider@arbmed. med.uni-giessen.de

\section{Zusammenfassung \\ $\nabla$}

Hintergrund: Arbeits- und sozialmedizinisch sind objektive Verfahren zur Abschätzung von Leistungseinschränkungen erforderlich. In der klinischen Arbeitsmedizin ist die spiroergometrische Belastungsuntersuchung etabliert. Sportphysiologisch sind Laktatbestimmungen üblich. Normwerte von arbeitsmedizinisch relevanten Leistungsdaten für ältere Probanden sind relevant, da Patienten in der Begutachtung überwiegend ein höheres Lebensalter aufweisen. Zur Ermittlung der altersentsprechenden Leistungsfähigkeit wurden gesunde Männer und Frauen im Alter von über 60 Jahren untersucht.

Methode und Patienten: 43 Personen $\left(21 \% / 22 \sigma^{\pi}\right)$ im Alter von 60 - 79 Jahren wurden spiroergometrisch untersucht und Laktat wurde gemessen. Die Ergebnisse wurden mit bisherigen Normwerten verglichen.

Ergebnisse: Die Bruttoleistungsfähigkeit in Watt (W) lag bei den Frauen im Mittel bei $98 \mathrm{~W}$, bei den Männern bei $155 \mathrm{~W}$ und fiel im Altersverlauf ab (o 60-69 Jahre 100W, 70 - 79 Jahre $93 \mathrm{~W}$; ơ 60-69 Jahre 165W, 70-79 Jahre 139W). Bei Frauen lag die maximale Sauerstoffaufnahme (o $1476 \pm 275$ $\mathrm{ml} / \mathrm{min}$, ơ $2292 \pm 245 \mathrm{ml} / \mathrm{min}$ ), die gewichtsbezogene Sauerstoffaufnahme ( $21,6 \pm 4,9 \mathrm{ml} / \mathrm{min} / \mathrm{kg}$, $\sigma^{x} 28,4 \pm 4,6 \mathrm{ml} / \mathrm{min} / \mathrm{kg}$ ) und der Sauerstoffpuls (o $10,7 \pm 2,8 \mathrm{ml}, o^{\star} 16,4 \pm 2,1 \mathrm{ml}$ ) signifikant niedriger als bei den Männern $(p<0,001)$. Das maximal erreichte Laktat lag bei den Frauen im Mittel bei 5,83 $\mathrm{mmol} / \mathrm{l}$, bei den Männern bei $6,58 \mathrm{mmol} / \mathrm{l}(\mathrm{p}=$ $0,76)$.

Schlussfolgerungen: In vorhandenen Studien zur Bestimmung von Normwerten wurden die für die Abschätzung von Leistungseinschränkungen erforderlichen leistungsphysiologischen Parameter überwiegend an jüngeren Personen erhoben. Die leistungsphysiologischen Parameter können nicht auf ältere Personen bezogen werden, da diese im höheren Lebensalter deutlich niedriger liegen. Bei

\section{Abstract \\ $\nabla$}

Background: In order to determine physical capability, objective methods for the assessment of performance are required. Spiroergometry is well established in occupational and social medicine. The existing standard values are defined for younger adults; however patients have predominantly an older age. To determine the age-related physical capability healthy men and women at the age over 60 years were studied.

Methods and Patients: 43 persons $\left(21 \% / 22 \sigma^{\pi}\right)$, aged 60-79 years were examined spiroergometrically, including lactate measurements. The results were compared with previously published standard values.

Results: The maximum capacity in watts (W) for women was $98 \mathrm{~W}$, and for men $155 \mathrm{~W}$ and declined with increasing age ( $\circ 60-69$ years $100 \mathrm{~W}$, o 70-79 years $93 \mathrm{~W}$; $0^{x} 60-69$ years $165 \mathrm{~W}$. $\sigma^{7}$ $70-79$ years $139 \mathrm{~W}$ ). The maximum oxygen uptake (o $1476 \pm 275 \mathrm{~mL} / \mathrm{min}$, o $^{\pi} 2292 \pm 245 \mathrm{~mL} / \mathrm{min}$ ), the weight-specific oxygen uptake (o $21.6 \pm 4.9$ $\mathrm{mL} / \mathrm{kg} / \mathrm{min}, o^{7} 28.4 \pm 4.6 \mathrm{~mL} / \mathrm{kg} / \mathrm{min}$ ) and the oxygen pulse ( $q 10.7 \pm 2.8 \mathrm{~mL}, \sigma^{\star} 16.4 \pm 2.1 \mathrm{~mL}$ ) were significantly lower in woman compared to men $(\mathrm{p}<0.001)$. The maximum lactate was achieved in women with a mean value of $5.83 \mathrm{mmol} / \mathrm{L}$, and for men of $6.58 \mathrm{mmol} / \mathrm{L}(\mathrm{p}=0.76)$.

Conclusions: Studies for the collection of normal values have mainly involved young people. The physiological performance parameters cannot be referred to elderly persons. In the socio-medical assessment age-related normative values should be used to determine residual capacity.

\footnotetext{
* Teile der Ergebnisse sind Bestandteile der Doktorarbeit von Frau M. Funk.
} 
der Ermittlung von Leistungseinschränkungen in der sozialmedizinischen Begutachtung sollten die altersentsprechenden Referenzwerte zugrunde gelegt werden.

\section{Einleitung}

Arbeits- und sozialmedizinisch haben Verfahren zur Abschätzung der Leistungseinschränkung bei Berufskrankheiten und Erwerbsminderungsrenten einen großen Stellenwert. In der Gesetzlichen Unfallversicherung wird die Minderung der Erwerbsfähigkeit (MdE) ermittelt. Bei den Rentenversicherungsträgern erfolgt die Abschätzung des noch vorhandenen Restleistungsvermögens als Kriterium für den Bezug einer vollen oder halben Erwerbsminderungsrente (VEMR/HEMR). Die Ermittlung der Rest-Leistungsfähigkeit ist relevant für den Grad der Beeinträchtigung zur Gewährung von Leistungen bei einer Berufsunfähigkeit. Arbeitsund sozialmedizinisch sind daher objektive Verfahren zur Abschätzung von Leistungseinschränkungen erforderlich. Beginnende Leistungseinschränkungen werden im Allgemeinen erst bei körperlichen Belastungen manifest. Zur Bestimmung der kardiopulmonalen Leistungsfähigkeit hat sich daher in der klinischen Arbeitsmedizin die spiroergometrische Belastungsuntersuchung etabliert [1]. Bei der Begutachtung unter den obengenannten Fragestellungen ist nicht die kurzzeitig mögliche Spitzenleistung ausschlaggebend, vielmehr muss eine Aussage hinsichtlich der über die Dauer einer Arbeitsschicht, also im Allgemeinen etwa acht Stunden, erbringbaren Leistung getroffen werden. Bei der Spiroergometrie lassen sich über die Bestimmung der anaeroben Schwelle mittels der Respiratory Exchange Rate (RER) Aussagen über die Dauerleistungsfähigkeit machen [2]. Der RER ist allerdings abhängig von der Ernährung, als auch willentlich etwa durch Hyperventilation beeinflussbar. Daher ist es ergänzend sinnvoll, die anaerobe Schwelle als Kriterium der Dauerleistungsfähigkeit auch metabolisch mittels Laktatkonzentrationsanstieg während der Belastungsuntersuchung zu bestimmen [3]. Neben der Bestimmung der Bruttoleistungsfähigkeit sind weitere spiroergometrische Kriterien der Leistungsbeurteilung wie Ermittlung der maximalen Sauerstoffaufnahme, die Sauerstoffaufnahme im Verhältnis zum Körpergewicht und der Sauerstoffpuls erforderlich. Vorhandene Studien zur Ermittlung von Normwerten haben die für die Abschätzung von Leistungseinschränkungen erforderlichen leistungsphysiologischen Parameter jedoch überwiegend bei jüngeren Personen erhoben. Ermittlungen von Normwerten für ältere Erwachsene stützen sich oft auf kleinere Fallzahlen. Zudem sind die leistungsphysiologisch relevanten Parameter meist unvollständig erhoben worden. Patienten in der arbeits- und sozialmedizinischen Begutachtung weisen jedoch überwiegend ein höheres Lebensalter auf. Zur Ermittlung der altersentsprechenden Referenzwerte wurden in der vorliegenden Studie gesunde Männer und Frauen über 60 Jahre untersucht.
Zusätzlich erfolgte der Vergleich der ermittelten Werte mit den in der Literatur bisher publizierten Normwerten.

\section{Kollektiv}

Die Probanden wurden aus der lokalen Bevölkerung requiriert. Eingeschlossen wurden im normalen Umfang körperlich aktive, gesunde Erwachsene über 60 Jahre. Ausgeschlossen wurden Personen mit Erkrankungen, die sich auf die allgemeine Leistungsfähigkeit auswirken wie koronare Herzkrankheit (KHK), hypertensive Herzkrankheit sowie Atemwegs- und Lungenkrankheiten mit obstruktiven oder restriktiven Ventilationsstörungen. Ebenfalls ausgeschlossen wurden Personen, die regelmäßig Ausdauertraining von mehr als 3 Stunden Dauer pro Woche absolvieren, aktive Leistungssportler und Patienten mit einer anerkannten Berufskrankheit. Kein Ausschlusskriterium war eine ausreichend behandelte Hypertonie. Dieser Faktor wurde gezielt auf seinen Einfluss auf die erhobenen Befunde geprüft.

Die Ethik-Kommission am Fachbereich Medizin der Justus-Liebig Universität in Gießen genehmigte die Studie (Az.: 251/11).

Von allen potenziellen Teilnehmern wurde eine ausführliche Anamnese erhoben und eine klinische Untersuchung einschließlich Ruhe-Elektrokardiogramm, Blutdruckmessung und Lungenfunktionsprüfung durchgeführt. Untersucht wurden zunächst 52 Personen, hiervon 24 Frauen und 28 Männer. Neun Personen wurden im Nachhinein ausgeschlossen. Eine Frau hatte eine bereits seit den 80er-Jahren anerkannte Berufskrankheit, eine litt an einem akuten Infekt, drei Probanden an einer KHK und einer an einem Asthma bronchiale. Bei drei Probanden wurde die spiroergometrische Untersuchung vorzeitig wegen Herzrhythmusstörungen, überschießenden Blutdrucks bzw. orthopädischer Beschwerden abgebrochen. Letztlich konnten 43 Personen (21 o/ $22 \sigma^{\pi}$ ) im Alter von 60-79 Jahren in die Studie aufgenommen werden.

Das Durchschnittsalter war bei den Frauen im Mittel mit $66 \pm 4,7$ Jahren vergleichbar mit den Männern mit $67 \pm 5,1$ Jahren (siehe - Tab.1). Das Gewicht der Frauen lag im Mittel mit 69,2 $\pm 4,7$ Kilogramm signifikant niedriger als bei den Männern mit 81,6 \pm $10,5 \mathrm{~kg}(\mathrm{p}<0,001)$. Der Broca-Index bei Frauen $(108 \pm 9,8 \%)$ unterschied sich nicht von den Männern $(107 \pm 8,1 \%)(p=0,79)$. Auch in den Altersgruppen der 60- bis 69-Jährigen und der 70bis 79-Jährigen fanden sich bezüglich des Broca-Index keine signifikanten Änderungen. Es fand sich kein aktiver Zigarettenraucher unter den untersuchten Probanden. Lediglich ein Studienteilnehmer gab einen Rauchkonsum von 2-3 Pfeifen am Abend an.

Tab. 1 Anthropometrische Daten der Frauen und Männer nach Altersklassen. Angegeben ist der Mittelwert und die Standardabweichung.

\begin{tabular}{|c|c|c|c|c|c|c|}
\hline \multirow[b]{2}{*}{ Parameter (Einheit) } & \multicolumn{3}{|l|}{ Frauen } & \multicolumn{3}{|l|}{ Männer } \\
\hline & Gesamt & 60-69 Jahre & 70-79 Jahre & Gesamt & 60-69 Jahre & 70-79 Jahre \\
\hline Alter (Jahre) & $66 \pm 4,7$ & $64 \pm 2,6$ & $72 \pm 2,5$ & $67 \pm 5,0$ & $64 \pm 2,4$ & $73 \pm 3,0$ \\
\hline Gewicht (kg) & $69,2 \pm 4,7$ & $68 \pm 5,8$ & $72 \pm 7,9$ & $81,6 \pm 10,5$ & $84 \pm 11$ & $79 \pm 9$ \\
\hline Broca (\%) & $108 \pm 9,8$ & $106 \pm 7,4$ & $113 \pm 12,7$ & $107 \pm 8,1$ & $109 \pm 11$ & $107 \pm 7$ \\
\hline
\end{tabular}


Tab. 2 Spiroergometrische Parameter bei Männern und Frauen nach Altersklassen. Angegeben ist der Mittelwert und die Standardabweichung.

\begin{tabular}{|c|c|c|c|c|c|c|}
\hline & Frauen & & & Männer & & \\
\hline Parameter (Einheit) & Gesamt & 60-69 Jahre & 70-79 Jahre & Gesamt & 60-69 Jahre & 70-79 Jahre \\
\hline Anzahl (Personen) & 21 & 14 & 7 & 22 & 13 & 9 \\
\hline $\begin{array}{l}\text { Leistung } \\
\text { (Watt) }\end{array}$ & $98 \pm 16$ & $100 \pm 17$ & $93 \pm 12$ & $155 \pm 26$ & $165 \pm 22$ & $139 \pm 25$ \\
\hline $\begin{array}{l}\text { Leistung } \\
\text { (Watt/kgKG) }\end{array}$ & $1,43 \pm 0,23$ & $1,47 \pm 0,23$ & $1,33 \pm 0,22$ & $1,92 \pm 0,46$ & $2,03 \pm 0,49$ & $1,77 \pm 0,37$ \\
\hline RER max & $1,08 \pm 0,08$ & $1,09 \pm 0,08$ & $1,05 \pm 0,07$ & $1,07 \pm 0,15$ & $1,05 \pm 0,08$ & $1,12 \pm 0,22$ \\
\hline $\begin{array}{l}\text { Laktat } \\
(\mathrm{mmol} / \mathrm{l})\end{array}$ & $5,83 \pm 1,52$ & $6,14 \pm 1,61$ & $5,21 \pm 1,21$ & $6,58 \pm 2,77$ & $7,15 \pm 3,21$ & $5,77 \pm 1,86$ \\
\hline $\begin{array}{l}\mathrm{VO}_{2} \max \\
(\mathrm{ml} / \mathrm{min})\end{array}$ & $1476 \pm 275$ & $1431 \pm 254$ & $1566 \pm 313$ & $2292 \pm 245$ & $2402 \pm 189$ & $2134 \pm 237$ \\
\hline $\begin{array}{l}\mathrm{VO}_{2} \mathrm{AT} \\
(\mathrm{ml} / \mathrm{min})\end{array}$ & $1363 \pm 287$ & $1334 \pm 279$ & $1455 \pm 294$ & $1898 \pm 308$ & $1901 \pm 343$ & $1892 \pm 246$ \\
\hline $\begin{array}{l}\mathrm{VO}_{2} \max / \mathrm{kg} \\
(\mathrm{ml} / \mathrm{kg} / \mathrm{min})\end{array}$ & $21,6 \pm 4,9$ & $21,2 \pm 4,3$ & $22,3 \pm 6,3$ & $28,4 \pm 4,6$ & $29,2 \pm 4,7$ & $27,4 \pm 4,5$ \\
\hline $\begin{array}{l}\mathrm{VO}_{2} \mathrm{AT} / \mathrm{kg} \\
(\mathrm{ml} / \mathrm{kg} / \mathrm{min})\end{array}$ & $20,0 \pm 5,1$ & $19,7 \pm 4,5$ & $20,8 \pm 6,6$ & $23,6 \pm 6,0$ & $23,8 \pm 7,2$ & $23,1 \pm 2,0$ \\
\hline $\begin{array}{l}\mathrm{O}_{2} \text { Puls max } \\
\text { (ml/Schlag) }\end{array}$ & $10,7 \pm 2,8$ & $9,8 \pm 1,8$ & $12,3 \pm 3,7$ & $16,4 \pm 2,1$ & $16,2 \pm 1,9$ & $16,6 \pm 2,5$ \\
\hline $\begin{array}{l}\mathrm{AaDO}_{2} \\
(\mathrm{mmHg})\end{array}$ & $19 \pm 7$ & $19 \pm 7$ & $21 \pm 7$ & $17 \pm 8$ & $19 \pm 8$ & $12 \pm 6$ \\
\hline $\begin{array}{l}\mathrm{pO}_{2} \text { Ruhe } \\
(\mathrm{mmHg})\end{array}$ & $81,4 \pm 8,7$ & $82,4 \pm 9,8$ & $79,3 \pm 6,3$ & $83,4 \pm 7,8$ & $82,0 \pm 7,0$ & $85,7 \pm 8,9$ \\
\hline $\begin{array}{l}\mathrm{pO}_{2} \text { unter Belastung } \\
(\mathrm{mmHg})\end{array}$ & $92,6 \pm 7,9$ & $94,0 \pm 7,3$ & $89,7 \pm 8,7$ & $98,7 \pm 10,5$ & $97,6 \pm 8,3$ & $98,9 \pm 13,6$ \\
\hline $\begin{array}{l}\mathrm{pCO}_{2} \text { Ruhe } \\
(\mathrm{mmHg})\end{array}$ & $34,8 \pm 4,8$ & $35,3 \pm 4,5$ & $34,0 \pm 5,8$ & $34,9 \pm 3,2$ & $34,5 \pm 3,2$ & $35,6 \pm 3,4$ \\
\hline $\begin{array}{l}\mathrm{pCO}_{2} \text { unter Belastung } \\
(\mathrm{mmHq})\end{array}$ & $35,0 \pm 4,8$ & $34,4 \pm 5,2$ & $36,2 \pm 4,2$ & $30,4 \pm 3,9$ & $29,6 \pm 4,0$ & $31,9 \pm 3,6$ \\
\hline
\end{tabular}

AT = Anaerobic Threshold (anaerobe Schwelle).

\section{Methoden}

Von allen Studienteilnehmern wurde eine ausführliche Anamnese erhoben und eine klinische Untersuchung einschließlich Ruhe-Elektrokardiogramm und Lungenfunktionsprüfungen durchgeführt. Alle Probanden wurden spirometrisch mit dem „Oxycon Alpha“ auf einem Fahrradergometer Typ „ER 900“ der Firma Jaeger/Viasys, Höchberg, untersucht. Die Belastung erfolgte im Sitzen, beginnend mit 25 bzw. 50 Watt und Steigerung um 25 Watt alle 3 Minuten. Es wurde ein 12-Kanal-EKG registriert und der Blutdruck in Ruhe und unter Belastung gemessen. Spirometrisch wurden das Atemminutenvolumen, die Atemfrequenz, die Sauerstoffaufnahme, die $\mathrm{CO}_{2}$-Abgabe, das maximal ventilierte Volumen und der RER bestimmt. Hauptzielkriterien waren die Bruttoleistungsfähigkeit in Watt (W), die körpergewichtsbezogene maximale Leistungsfähigkeit in Watt pro Kilogramm Körpergewicht (Watt $/ \mathrm{kgKG})$, die maximale Sauerstoffaufnahme $(\mathrm{ml} /$ min) sowie die körpergewichtsbezogene Sauerstoffaufnahme $(\mathrm{ml} / \mathrm{kgKG} / \mathrm{min})$ und der Sauerstoffpuls $\left(\mathrm{O}_{2}\right.$-Aufnahme in $\mathrm{ml}$ pro Herzrate). Außerdem sollte die Dauerleistungsgrenze mittels des respiratorischen Quotienten und der Laktatschwelle bestimmt werden.

Zur Laktat- und Blutgasbestimmung wurde in Ruhe und jeweils am Ende der 3-minütigen Belastungsstufen Kapillarblut aus dem hyperämisierten Ohrläppchen entnommen. Sofern keine für die Ergometrie allgemeingültigen Abbruchkriterien eintraten, wurde der Proband bis zur subjektiven Erschöpfung belastet.

\section{Statistik}

Der statistisch-biometrische Gruppenvergleich erfolgte mit dem parameterfreien Wilcoxon-Test für unverbundene Stichproben. Die lineare Regression mit Ermittlung des Rangkorrelationskoeffizienten ( $r$ ) nach Spearman als auch die multiplen logistischen Regressionsanalysen erfolgten unter Verwendung von SPSS 19.0. P-Werte $<0,05$ wurden als ein signifikantes Ergebnis angesehen.

\section{Ergebnisse}

Die Bruttoleistungsfähigkeit in Watt lag bei den Frauen im Mittel bei $98 \pm 16 \mathrm{~W}$ und somit signifikant niedriger als bei den Männern mit $155 \pm 26 \mathrm{~W}(\mathrm{p}<0,001)$. Die Leistungsfähigkeit sowohl bei Männern als auch bei Frauen fiel im höheren Alter ab. Die Männer erbrachten im Alter von 60-69 Jahren im Mittel $165 \pm 22 \mathrm{~W}$, im Alter von 70-79 Jahren $139 \pm 25 \mathrm{~W}(\mathrm{p}<0,05)$, siehe $\bullet$ Tab. 2 . Sie lag bei den Frauen im Alter von $60-69$ Jahren bei $100 \pm 17 \mathrm{~W}$ und bei den über 70 -jährigen mit $93 \pm 12 \mathrm{~W}$ niedriger $(\mathrm{p}=0,44)$. Die Altersabhängigkeit der Bruttoleistungsfähigkeit wurde weiterhin mithilfe von Korrelationsgrafiken unter Angabe des 90\%-Konfidenzintervalls grafisch dargestellt, siehe $\boldsymbol{\bullet}$ Abb.1. Es ergaben sich folgende Regressionsgleichungen für Männer (W): $-3,19 \times$ Alter (in Jahren) $+371,17$ und für Frauen $(W)$ : $-0,37 \times$ Alter (in Jahren) $+122,17$. Für Männer wurde der Korrelationskoeffizient (r) mit $r=-0,62$, für Frauen mit $r=-0,10$ errechnet. Sowohl in der jüngeren als auch in der älteren Gruppe bestanden hochsignifikante Unterschiede in der Bruttoleistungsfähigkeit in Watt 


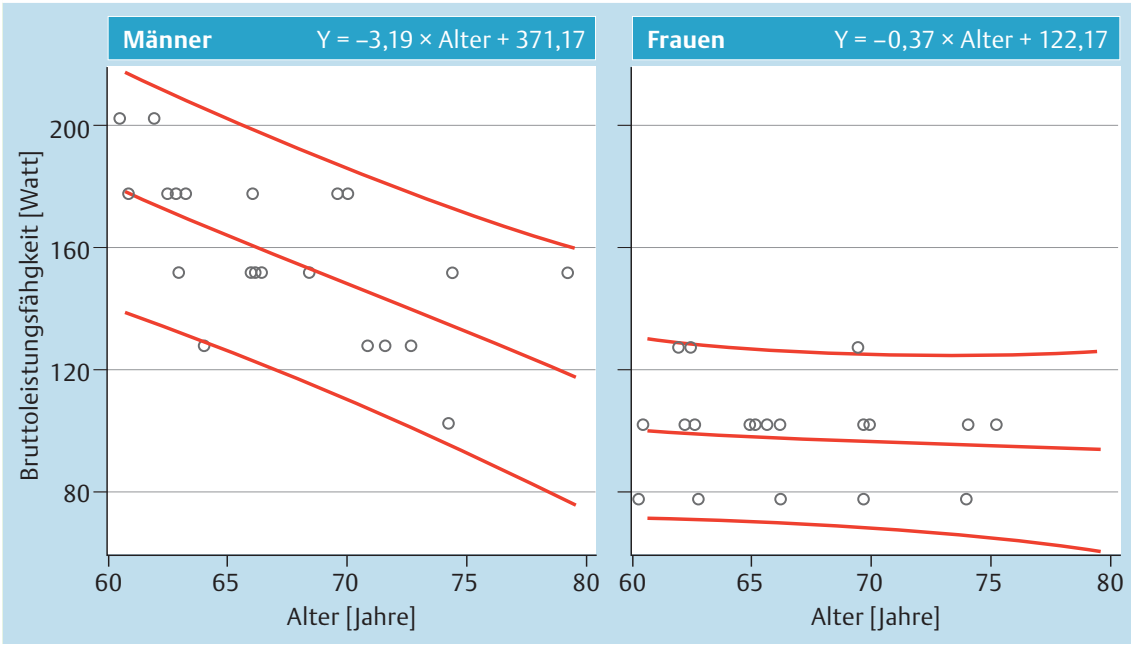

Abb. 1 Korrelation der Bruttoleistungsfähigkeit mit dem Lebensalter zwischen 60 und 80 Jahren. Eingetragen sind die lineare Regression und das $90 \%$-Konfidenzintervall.

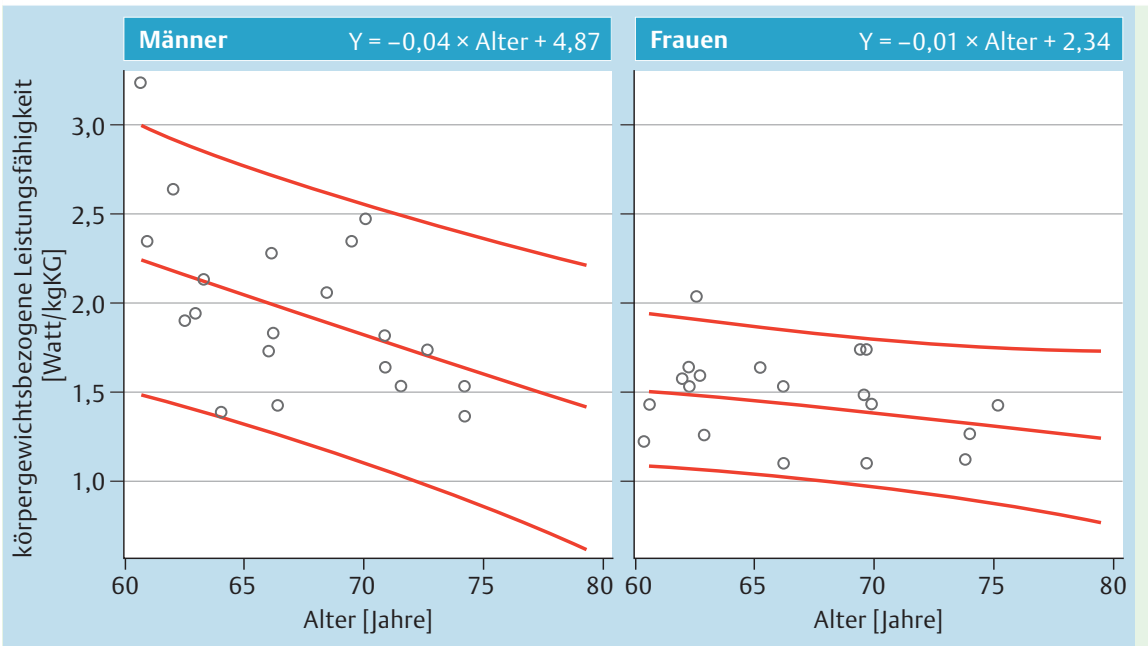

Abb. 2 Korrelation der körpergewichtsbezogenen Leistungsfähigkeit mit dem Lebensalter zwischen 60 und 80 Jahren. Eingetragen sind die lineare Regression und das $90 \%$-Konfidenzintervall.

zwischen den Geschlechtern ( $\mathrm{p}=0,001$ bei den Jüngeren bzw. $\mathrm{p}<$ 0,001 bei den Älteren). Die multiplen logistischen Regressionsanalysen zeigten, dass das Gewicht oder der Broca-Index keinen signifikanten Einfluss hatten auf die Bruttoleistungsfähigkeit. Ebenfalls hochsignifikant war der Unterschied in der erreichten Leistung in Watt, bezogen auf das Körpergewicht (KG) im Vergleich von Männern und Frauen. In der Gruppe der 60- bis 69Jährigen erbrachten die Frauen eine Leistung von im Mittel 1,47 $\pm 0,23 \mathrm{~W} / \mathrm{kgKG}$, die Männer von 2,03 $\pm 0,49 \mathrm{~W} / \mathrm{kgKG}(\mathrm{p}=0,001)$. Bei den Älteren erbrachten die Frauen eine Leistung von 1,33 \pm $0,22 \mathrm{~W} / \mathrm{kgKG}$, die Männer von 1,77 $\pm 0,37 \mathrm{~W} / \operatorname{kgKG}(\mathrm{p}=0,008)$. Die körpergewichtsbezogene Leistungsfähigkeit war bei beiden Geschlechtern im höheren Lebensalter niedriger, siehe $\bullet$ Abb. 2 . Es ergaben sich folgende Regressionsgleichungen für Männer (W/kgKG): $-0,04 \times$ Alter (in Jahren) $+4,87$, und für Frauen (W/ $\mathrm{kgKG}$ ): $-0,01 \times$ Alter (in Jahren) $+2,34$. Die reduzierte körpergewichtsbezogene Leistungsfähigkeit in $\mathrm{W} / \mathrm{kgKG}$ in den höheren Altersgruppen war jedoch nicht signifikant (Frauen $\mathrm{p}=0,26$, Männer $\mathrm{p}=0,16)$. Für Männer wurde der Korrelationskoeffizient (r) mit r = -0,48, für Frauen mit $r=-0,26$ errechnet.

Bei Frauen lag die maximale Sauerstoffaufnahme (Frauen: 1476 $\pm 275 \mathrm{ml} / \mathrm{min}$; Männer: $2292 \pm 245 \mathrm{ml} / \mathrm{min}$ ), die gewichtsbezogene Sauerstoffaufnahme (Frauen: $21,6 \pm 4,9 \mathrm{ml} / \mathrm{kg} / \mathrm{min}$, Männer: $28,4 \pm 4,6 \mathrm{ml} / \mathrm{kg} / \mathrm{min}$ ) und der Sauerstoffpuls (Frauen: 10,7 $\pm 2,8$ ml, Männer: $16,4 \pm 2,1 \mathrm{ml}$ ) jeweils signifikant niedriger als bei den Männern ( $p<0,001$ ), siehe Tab. 2. Bezogen auf das Alter er- gaben sich folgende Korrelationskoeffizienten: für die maximale Sauerstoffaufnahme Männer $r=-0,62$, Frauen $r=0,30$, für die körpergewichtsbezogene Sauerstoffaufnahme Männer $r=-0,40$, Frauen $r=0,10$ und für den Sauerstoffpuls Männer $r=0,20$, Frauen $r=0,37$. Die multiplen logistischen Regressionsanalysen zeigten, dass der Broca-Index keinen signifikanten Einfluss hatte.

Der maximal erreichte RER unterschied sich ebenfalls nicht signifikant; der Korrelationskoeffizient lag nahe 0.

Die anaerobe Schwelle wurde näherungsweise anhand eines RER von 1,0 bestimmt; sie dient als Beurteilungskriterium der Dauerleistungsfähigkeit und liegt demzufolge niedriger als die jeweiligen Maximalwerte. Die erreichte Leistung an der metabolisch bzw. respiratorisch bestimmten anaeroben Schwelle war bei den Frauen mit zunehmendem Alter konstant, jeweils $r=0,00$. Bei den Männern fiel die Bruttoleistung in Watt an der Schwelle mit einem RER von 1,0 nur leicht ab; für die respiratorische anaerobe Schwelle ergab sich $r=-0,20$, an der metabolischen Schwelle $r=-0,27$.

Auch an der anaeroben Schwelle wurden die spirometrischen Parameter im Altersverlauf ermittelt. Es ergab sich für die Sauerstoffaufnahme an der anaeroben Schwelle bei Männern ein Korrelationskoeffizient von $r=0,00$, bei Frauen $r=0,32$, für die körpergewichtsbezogene Sauerstoffaufnahme entsprechend $r=0,00$ und $r=0,14$, für den Sauerstoffpuls $r=0,58$ bzw. $r=0,52$. 
Die Blutgase vor und nach Belastung wurden ebenfalls geprüft. Der Korrelationskoeffizient für den Sauerstoffpartialdruck vor Belastung wurde bei Männern mit $r=0,25$, bei Frauen mit $r=$ $-0,10$ bestimmt; der Kohlendioxidpartialdruck vor Belastung mit $r=0,22$ bzw. $r=-0,14$. Nach Belastung ergaben sich folgende Korrelationskoeffizienten für den Sauerstoffpartialdruck $r=0,14$ bzw. $r=-0,14$ und den Kohlendioxidpartialdruck $r=0,22$ bzw. $r=0,17$.

Für die alveolär-arterielle Sauerstoffdifferenz fand sich keine Änderung im Altersverlauf bei den Frauen, während diese bei den Männern leicht abfiel $r=-0,37$.

Das maximal erreichte Laktat lag bei den Frauen im Mittel bei $5,83 \mathrm{mmol} / \mathrm{l}$, bei den Männern bei $6,58 \mathrm{mmol} / \mathrm{l}(\mathrm{p}=0,76)$. Es bestanden jeweils keine signifikanten Unterschiede zwischen den gemessenen maximalen Laktatwerten in den Altersgruppen (Männer $r=-0,62$, Frauen $r=-0,14$ ). Auch beim Vergleich der Geschlechtergruppen gleichen Alters ergaben sich keine signifikanten Unterschiede.

Die jeweils ermittelten Werte sind in $\bullet$ Tab. 2 zusammengestellt.

\section{Diskussion}

Unser Ziel war es, die für eine adäquate Beurteilung in sozial- und arbeitsmedizinischen Begutachtungen erforderlichen Parameter vollständig an einem definierten Kollektiv zu bestimmen. Die bisher publizierten Studien werden im Folgenden analysiert und mit den von uns ermittelten Werten verglichen. Normwerte von arbeitsmedizinisch relevanten Leistungsdaten für ältere Probanden sind relevant, da Patienten in der Begutachtung überwiegend ein höheres Lebensalter aufweisen. Normwerte sind meist an jüngeren Erwachsenen erhoben worden Die Anzahl an Studien bei älteren Personen ist überschaubar. Es fehlen aber Studien mit vollständiger Darstellung aller relevanten Parameter an einer Personengruppe im höheren Lebensalter.

\section{Die Beurteilung der Bruttoleistungsfähigkeit}

Bei nur drei Studien fand sich eine Mitteilung über die maximale Leistungsfähigkeit in Watt bei Personen in höherem Lebensalter. Die Bruttoleistungsfähigkeit von Männern und Frauen mit einem Durchschnittsalter von 66 Jahren lag bei $175 \pm 36$ W bzw. $102 \pm$ $25 \mathrm{~W}$ (Original-Daten in $\mathrm{kpm} / \mathrm{min} .1 \mathrm{~W}=6,12 \mathrm{kpm} / \mathrm{min}$ ) [4]. Pothoff et al. [5] untersuchten 116 Männer und Frauen im Altersverlauf. In der Altersgruppe von 60-69 Jahren wurden lediglich 10 Männer und 11 Frauen aufgenommen. Die Bruttoleistungsfähigkeit wurde bei den Männern mit $164 \pm 18 \mathrm{~W}$, bei Frauen mit $111 \pm 23 \mathrm{~W}$ bestimmt [5]. Vergleichbare Daten mit 156,1 $\pm 38,1 \mathrm{~W}$ an 47 Männern und $99,3 \pm 24,4 \mathrm{~W}$ an 48 Frauen berichteten Ong et al. [6]. Allerdings wurden erwachsene Chinesen mit einem Durchschnittsalter von 42 Jahren untersucht. In unserer eigenen Studie bestimmten wir die Bruttoleistungsfähigkeit bei Männern mit $155 \pm 26$ W, bei Frauen mit $98 \pm 16$ W. Es zeigte sich eine reduzierte Leistungsfähigkeit der 70-jährigen im Vergleich zu den 60bis 69-jährigen Männern ( $139 \pm 25$ W vs. $165 \pm 22$ W). Hingegen wurde dies nicht bei den Frauen beobachtet (60- bis 69-Jährige: $100 \pm 17 \mathrm{~W}$, über 70 -Jährige: $93 \pm 12 \mathrm{~W}$ ).

\section{Die körpergewichtsbezogene Leistungsfähigkeit}

Anstelle der Bruttoleistungsfähigkeit ist die Ermittlung der körpergewichtsbezogenen Leistungsfähigkeit in Watt aussagekräftiger, da hiermit die Leistungsfähigkeit von Personen mit unterschiedlichem Körperbau und Gewicht vergleichbar wird. Die

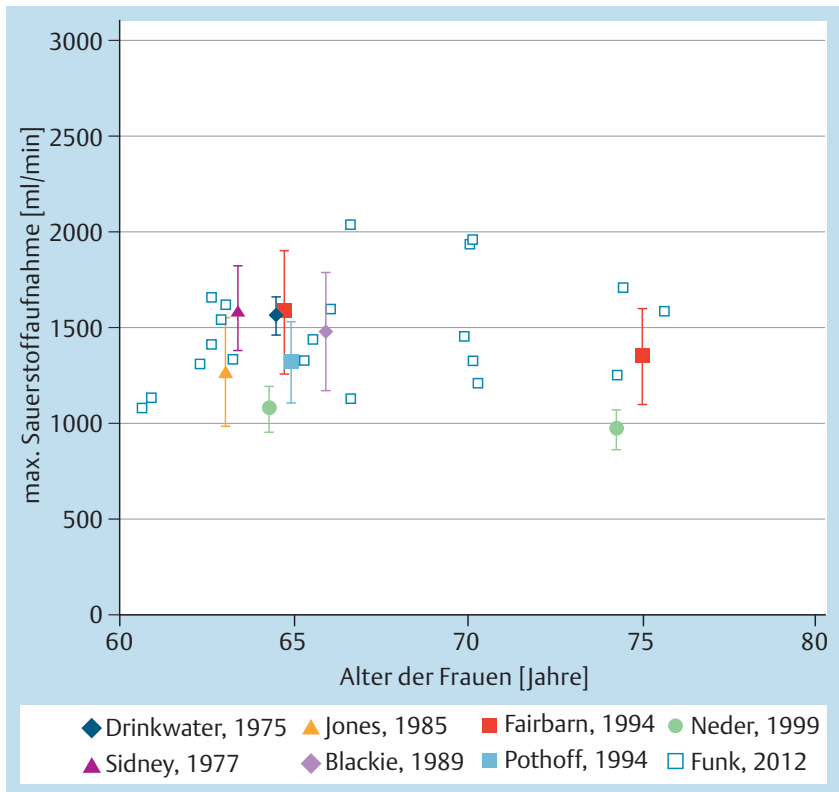

Abb.3 Maximale Sauerstoffaufnahme [in $\mathrm{ml} / \mathrm{min}$ ] bei Frauen im Alter über 60 Jahre. Dargestellt sind die eigenen Probandinnen als Punktwolke sowie die Mittelwerte und Standardabweichungen internationaler Studien. Bei Altersgruppen erfolgte der Eintrag in der Mitte.

gewichtsbezogene Leistungsfähigkeit in Watt pro Kilogramm (kg) Körpergewicht (KG) bestimmten wir bei den Männern mit $1,92 \pm 0,46 \mathrm{~W} / \mathrm{kgKG}$, bei den Frauen mit 1,43 $\pm 0,23 \mathrm{~W} / \mathrm{kgKG}$. Sie war niedriger bei den älteren Männern (60- bis 69-jährigen Männer: 2,03 $\pm 0,49 \mathrm{~W} / \mathrm{kgKG}$; über 70-jährige Männer: 1,77 $\pm 0,37 \mathrm{~W} /$ kgKG) und Frauen $(1,47 \pm 0,23 \mathrm{~W} / \mathrm{kgKG}$ vs. $1,33 \pm 0,22 \mathrm{~W} / \mathrm{kgKG})$. Die Angabe einer körpergewichtsbezogenen Leistungsfähigkeit in W/kgKG findet sich bei Sidney et al. [7]. Es wird die Physical Work Capacity (PWC) bei einer Herzfrequenz von 150 Schlägen pro Minute bezogen auf das Körpergewicht in Kilogramm (PWC $150 / \mathrm{kg}$ ) bei den Männern mit 1,59W/kgKG, bei den Frauen mit $1,32 \mathrm{~W} / \mathrm{kgKG}$ angegeben. Hierbei handelt es sich allerdings nicht um Daten der maximalen Belastbarkeit. Die maximale Leistungsfähigkeit in Watt, bezogen auf das Körpergewicht, konnten wir anhand der berichteten Daten aus der mittleren Bruttoleistungsfähigkeit und Standardabweichung in Watt und des Körpergewichtes annähernd errechnen [4-6]. Die körpergewichtsbezogene Leistungsfähigkeit für die über 60-jährigen Männer lag bei 2,36 $\pm 0,49 \mathrm{~W} / \mathrm{kgKG}$ bzw. 1,63 $\pm 0,40 \mathrm{~W} / \mathrm{kgKG}$ für Frauen [4], resp. bei 2,11 $\pm 0,23 \mathrm{~W} / \mathrm{kgKG}$ (Männer) und 1,65 $\pm 0,34 \mathrm{~W} / \mathrm{kgKG}$ (Frauen) [5]. Für Ong et al. [6] errechneten sich 2,32 $\pm 0,57 \mathrm{~W} / \mathrm{kgKG}$ (Männer) bzw. 1,80 $\pm 0,44 \mathrm{~W} / \mathrm{kgKG}$ (Frauen), wobei die untersuchten Personen jedoch ein deutlich jüngeres Durchschnittsalter von 42 Jahren aufwiesen.

\section{Die Ermittlung der maximalen Sauerstoffaufnahme} Hinsichtlich der maximalen Sauerstoffaufnahme (in $\mathrm{ml} / \mathrm{min}$ ) zeigte sich bei den untersuchten Frauen eine gute Übereinstimmung sämtlicher Studien [4,5,7-10], siehe Abb.3. Lediglich in einer Studie [11] lag die Sauerstoffaufnahme der Personen in den beiden Altersbereichen von 60 bis 69 sowie von 70 bis 79 Jahren unterhalb der Normalbereiche anderer Studien, wobei die Werte für eine Belastung mit $100 \mathrm{~W}$ angegeben werden (vgl. - Abb. 3). 


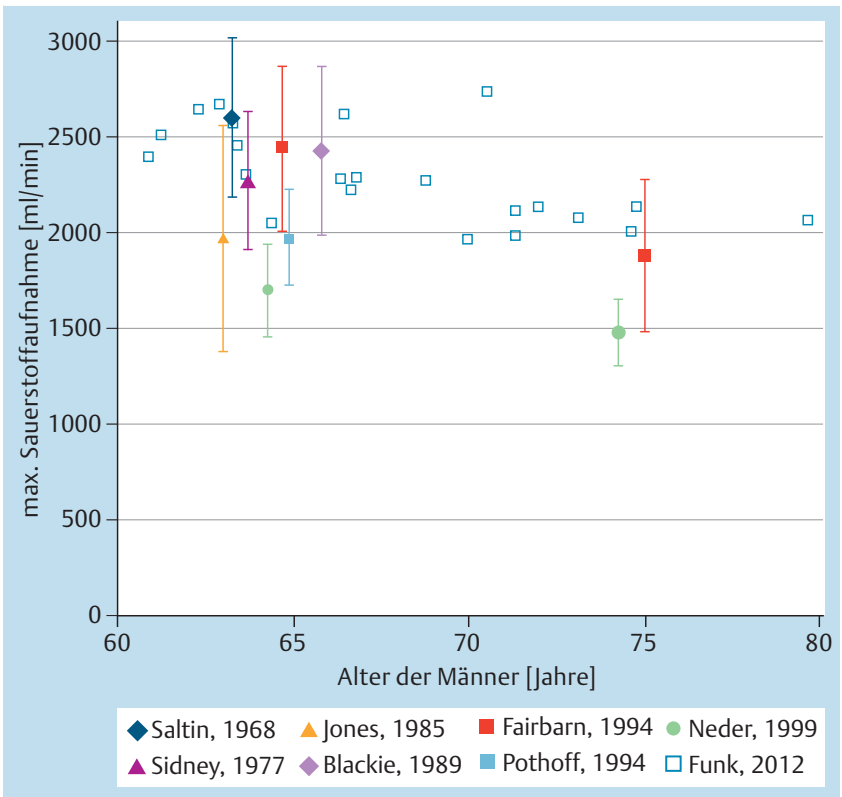

Abb. 4 Maximale Sauerstoffaufnahme [in $\mathrm{ml} / \mathrm{min}$ ] bei Männern. Legende siehe Abb. 3.

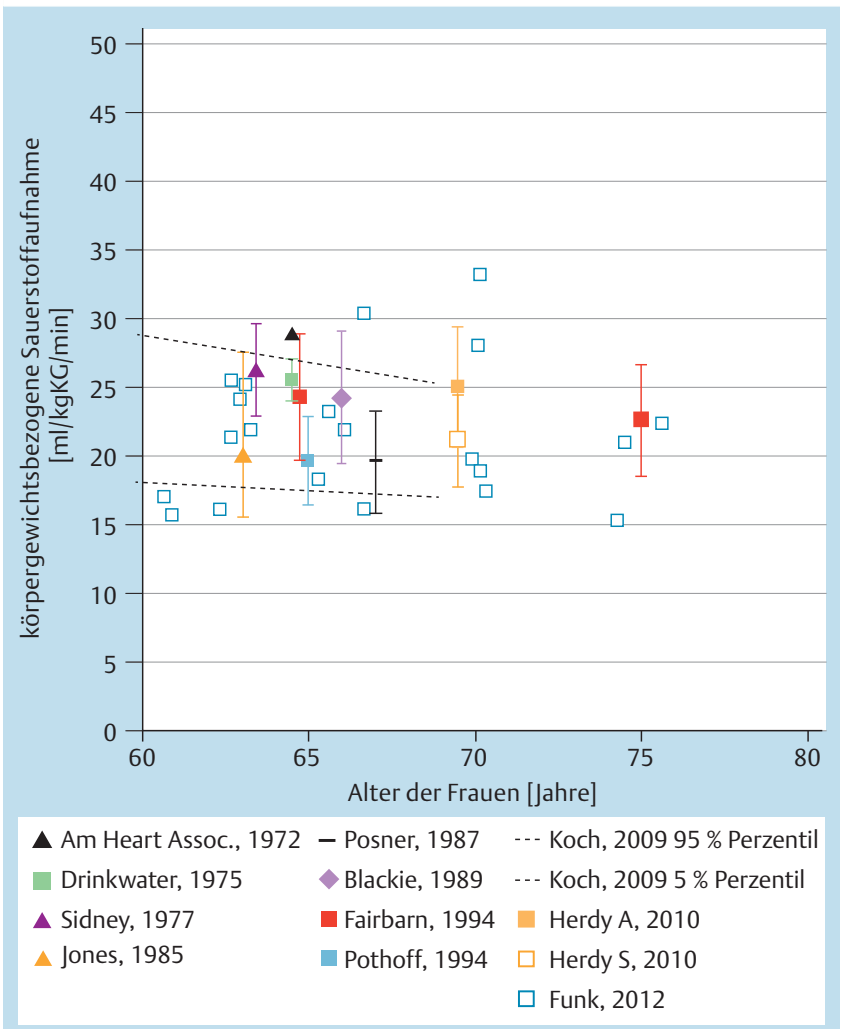

Abb.5 Maximale Sauerstoffaufnahme bezogen auf das Körpergewicht $[\mathrm{ml} / \mathrm{kg} / \mathrm{min}$ ] bei Frauen. $A=$ körperlich Aktive, $S=$ sitzende Tätigkeiten, Legende siehe Abb. $\mathbf{3}$.

Gleiches gilt auch für die maximale Sauerstoffaufnahme bei den Männern ( $\bullet$ Abb.4). Eine minütliche Steigerung der Belastung mittels Rampenprotokoll führten Pothoff et al. [5] und Neder et al. [11] durch, was mit einer teilweise deutlich niedrigeren maximalen Sauerstoffaufnahme einherging. Fairbarn et al. [10] führ-

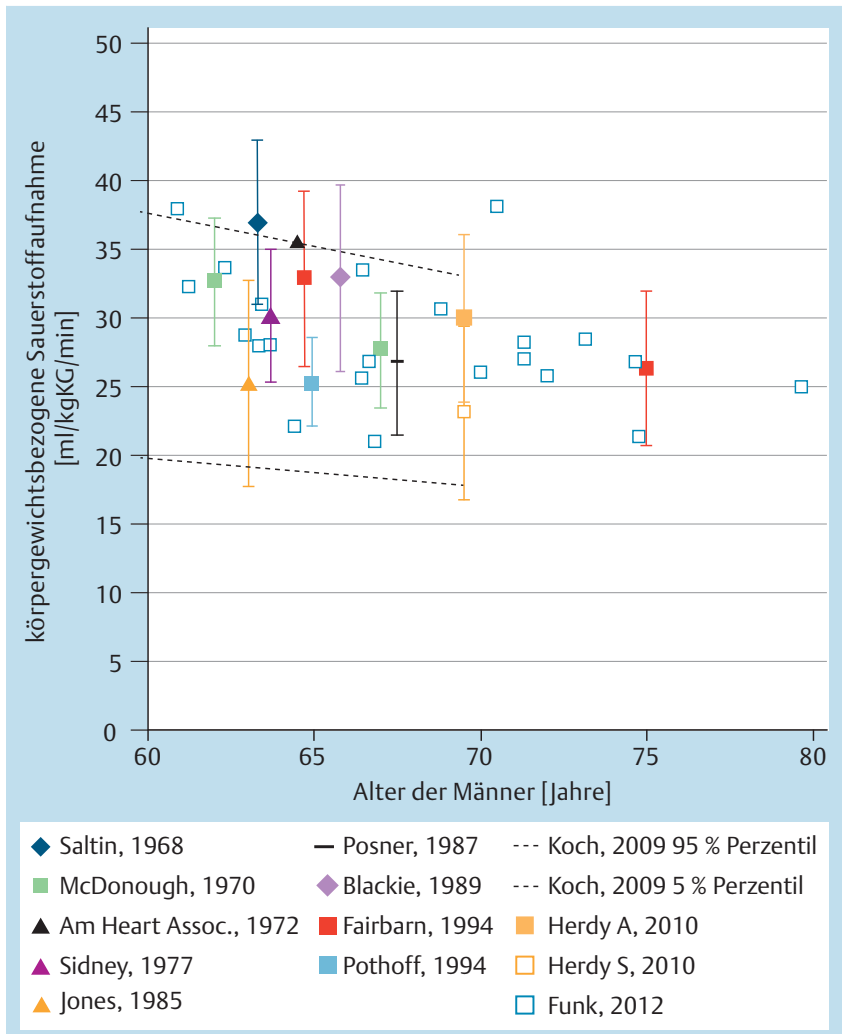

Abb.6 Maximale Sauerstoffaufnahme bezogen auf das Körpergewicht $[\mathrm{ml} / \mathrm{kg} / \mathrm{min}]$ bei Männern. Legende siehe Abb. 3 .

ten ebenfalls eine Ergometrie mit minütlicher Steigerung um $16 \mathrm{~W}$ durch. Eine gute Übereinstimmung fand sich zur Probandengruppe der 60- bis 69-Jährigen, während in der älteren Gruppe der 70- bis 80-Jährigen wie die von Neder et al. [11] berichteten Werte knapp unter den in unserer Studie ermittelten Werten der maximalen Sauerstoffaufnahme lagen. Jones et al. [9] belasteten sowohl 10 Männer als auch 10 Frauen im Alter von 55 bis 71 Jahren mit 300 bzw. $600 \mathrm{kpm} / \mathrm{min}$, also etwa 50 und 100 Watt. Es ergab sich eine relativ gute Übereinstimmung der maximalen Sauerstoffaufnahme bei Frauen $(1270 \pm 136 \mathrm{ml} / \mathrm{min})$. Die Sauerstoffaufnahme von Männern wird mit $1350 \pm 140 \mathrm{ml} / \mathrm{min}$ berichtet, wobei sich diese Angaben auf eine Belastung mit $100 \mathrm{~W}$ beziehen. So lag die Belastbarkeit der Männergruppe vergleichbaren Alters in unserer Studie mit 165W deutlich höher.

\section{Die auf das Körpergewicht bezogene maximale Sauerstoffaufnahme}

Zur inter- und intrapersonellen Vergleichbarkeit des Energieumsatzes insbesondere bei Tätigkeiten, bei denen die Beschäftigten ihr Körpergewicht wie beim Treppensteigen tragen müssen, ist die Ermittlung der körpergewichtsbezogenen Sauerstoffaufnahme von Bedeutung. Bezieht man daher die maximale Sauerstoffaufnahme (in ml/min) auf das Körpergewicht (kgKG) der Probanden, so finden sich bei den Frauen durchgängig in allen dargestellten Studien [4,5,7-10,12-15] gute Übereinstimmungen mit den von uns bestimmten Werten, vgl. $\bullet$ Abb.5. Diese Übereinstimmung ist unabhängig vom verwendeten Belastungsprotokoll. Gleiches gilt auch für die Studien an Männern [4,5, 7,9,10, 12-17], vgl. Abb. 6 . 


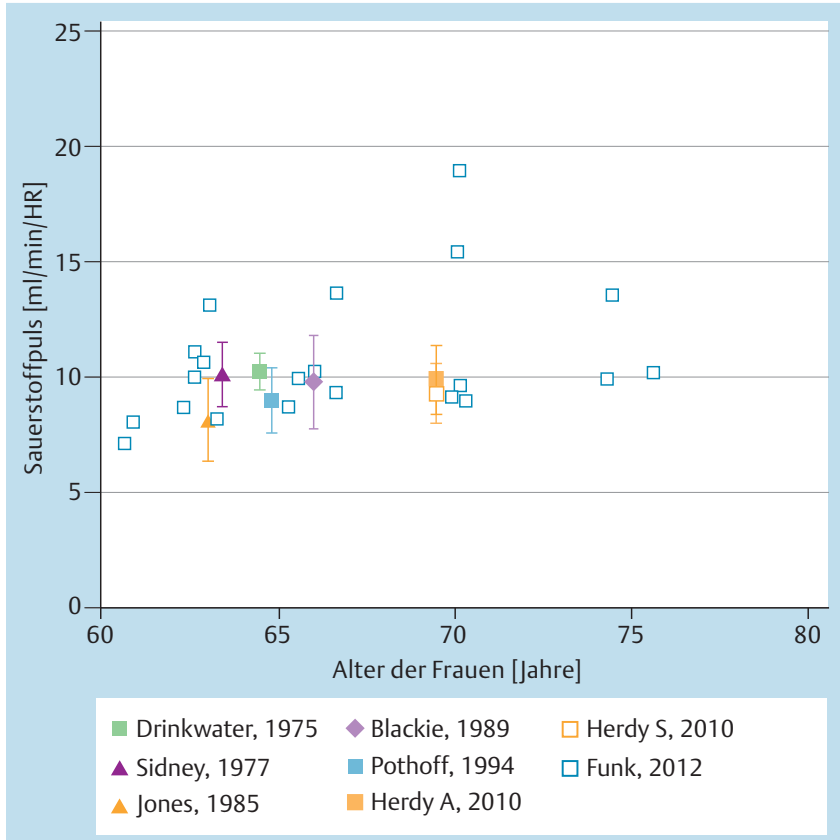

Abb.7 Maximaler Sauerstoffpuls [in $\mathrm{ml}$ ] bei Frauen. Legende siehe Abb. 3.

Die Sauerstoffaufnahme an der anaeroben Schwelle zur Beurteilung der Ausdauerleistungsfähigkeit

Zur Beurteilung der Ausdauerleistungsfähigkeit hat sich die Ermittlung der anaeroben Schwelle bei einer RER von 1,0 sportmedizinisch und arbeitsmedizinisch bewährt [18]. Die Ermittlung der Ausdauerleistungsfähigkeit ist sozialmedizinisch für die Beurteilung der körperlichen Belastbarkeit über die Dauer einer Arbeitsschicht relevant. Die Dauerleistungsgrenze liegt naturgemäß unter den Maximalwerten, vgl. $\bigcirc$ Tab.2. Lediglich bei zwei Studien kann der Parameter der Sauerstoffaufnahme an der anaeroben Schwelle als Dauerleistungsfähigkeit berechnet werden. Bei den Untersuchungen von Neder et al. [11] sowie Pothoff et al. [5] lag die Sauerstoffaufnahme an der anaeroben Schwelle sowohl von Männern $(887 \pm 125 \mathrm{ml} / \mathrm{min}$ resp. $1092 \pm$ $132 \mathrm{ml} / \mathrm{min})$ als auch von Frauen $(641 \pm 116 \mathrm{ml} / \mathrm{min}$ resp. $915 \pm$ $130 \mathrm{ml} / \mathrm{min}$ ) unterhalb der von uns ermittelten Werte (Männer: $1898 \pm 308 \mathrm{ml} / \mathrm{min}$, Frauen: $1363 \pm 287 \mathrm{ml} / \mathrm{min}$ ), welches auf das unterschiedliche Belastungsprotokoll mit minütlicher Steigerung der Belastung zurückgeführt wird.

\section{Die auf das Körpergewicht bezogene Sauerstoff-}

aufnahme an der anaeroben Schwelle

Der Sauerstoffaufnahme bezogen auf das Körpergewicht an der anaeroben Schwelle wird bei 55- bis 64-jährigen Männern (körperlich aktiv: $23,3 \pm 5,2$; wenig aktiv: $19,1 \pm 4,8 \mathrm{ml} / \mathrm{kg} / \mathrm{min}$ ) und Frauen (körperlich aktiv: 18,8 $\pm 4,4$; wenig aktiv: $16,1 \pm 2,8 \mathrm{ml} /$ $\mathrm{kg} / \mathrm{min}$ ) sowie bei 65 - bis 74 -jährigen Männern (körperlich aktiv: $19,9 \pm 4,8$; wenig aktiv: $15,9 \pm 4,5 \mathrm{ml} / \mathrm{kg} / \mathrm{min}$ ) und Frauen (körperlich aktiv: $17,4 \pm 3,1$; wenig aktiv: $14,9 \pm 2,9 \mathrm{ml} / \mathrm{kg} / \mathrm{min}$ ) angegeben [13].

Aus zwei Studien konnten die Daten der körpergewichtsbezogenen Sauerstoffaufnahme errechnet werden [5, 6]. An 42-Jährigen wurde die auf das Körpergewicht bezogene Sauerstoffaufnahme für Männer zu 14,8 $\pm 3,5 \mathrm{ml} / \mathrm{kg} / \mathrm{min}$, für Frauen zu 13,5 $\pm 3,33 \mathrm{ml} /$ $\mathrm{kg} / \mathrm{min}$ ermittelt [6]. Die korrespondierenden Werte bei Pothoff et al. [5] ergeben sich für 60- bis 69-jährige Männer zu 14,0 $\pm 1,7$

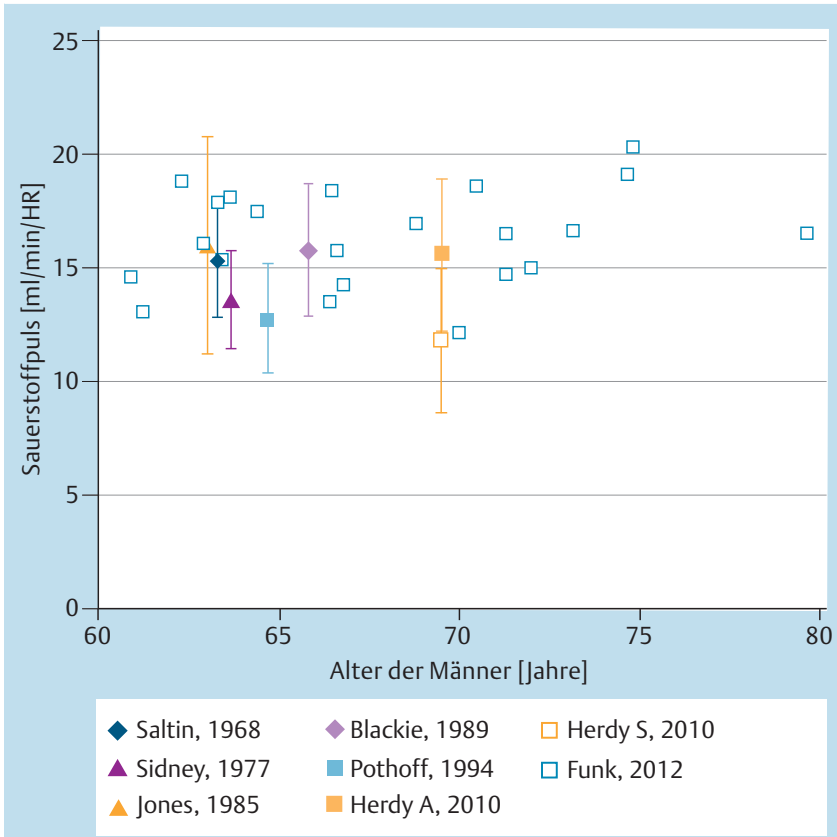

Abb.8 Maximaler Sauerstoffpuls [in $\mathrm{ml}]$ bei Männern. Legende siehe Abb. 3 .

und bei Frauen zu 13,7 $\pm 1,7 \mathrm{ml} / \mathrm{kg} / \mathrm{min}$. Weitere Daten konnten aus Originalabbildungen mittels einer Computersoftware ausgelesen werden $[12,19]$. Die körpergewichtsbezogene Sauerstoffaufnahme der 60 - bis 74 -Jährigen beträgt $15,7 \pm 2,7 \mathrm{ml} / \mathrm{kg} / \mathrm{min}$ und die der 75 - bis 89 -Jährigen $15,1 \pm 1,7 \mathrm{ml} / \mathrm{kg} / \mathrm{min}$ [12]. Nach Takeshima et al. [19] sind dies für die drei Altersgruppen 59-69, $70-79$ und $80-85$ Jahre bei Männern: $16,2 \pm 2,5 ; 14,4 \pm 2,9 ; 14,9$ $\pm 2,6$, bei Frauen: $24,0 \pm 4,2 ; 21,7 \pm 2,3$ und $21,5 \pm 1,7 \mathrm{ml} / \mathrm{kg} / \mathrm{min}$. Die in unserer Studie ermittelten körpergewichtsbezogenen Sauerstoffaufnahmen bei Männern $(23,6 \pm 6,0 \mathrm{ml} / \mathrm{kg} / \mathrm{min})$ entsprechen den körperlich aktiven Männern von Herdy et al. [13]. Für Frauen bestand eine Übereinstimmung mit Takeshima et al. [19]. Ansonsten lagen die körpergewichtsbezogenen Sauerstoffaufnahmen der Frauen mit durchschnittlich $20,0 \pm 5,1 \mathrm{ml} / \mathrm{kg} / \mathrm{min}$ oberhalb der in der Literatur berichteten Werte.

\section{Der maximale Sauerstoffpuls}

Der Sauerstoffpuls mit Standardabweichung wird in vier Studien angegeben $[5,8,13,20]$. Zusätzlich konnte der Sauerstoffpuls aus den Daten der maximalen Sauerstoffaufnahme, dividiert durch die maximale Herzfrequenz, annähend errechnet werden $[4,9$, $10,16,20]$.

Bei Frauen zeigt sich eine gute Übereinstimmung mit sämtlichen Studien $[4,5,7-10,13]$, siehe $\bullet$ Abb. 7 .

Bei den Männern liegt der von Pothoff et al. [5] und Herdy et al. [13] für inaktive Männer bestimmte Sauerstoffpuls unterhalb der eigenen sowie der in den übrigen Studien $[4,7,10,16]$ gefundenen Werte, vgl. $\bigcirc$ Abb. 8. In der älteren Gruppe der 65- bis 74Jährigen liegen die von Herdy et al. [13] untersuchten „Aktiven“ $(15,6 \pm 3,4 \mathrm{ml})$ im gleichen Bereich wie unsere 70 - bis 79-jährigen Probanden (16,6 $\pm 2,5 \mathrm{ml})$. Die von Fairbarn et al. [10] untersuchten 70- bis 80-jährigen Personen zeigten eine gute Übereinstimmung des Sauerstoffpulses $(12,29 \pm 2,6 \mathrm{ml})$ mit den 65 - bis 74 jährigen „Nichtaktiven“ (11,8 $\pm 3,2 \mathrm{ml})$ von Herdy et al. [13]. 
Maximal erreichte Respiratory Exchange Rate (RER)

Der maximal erreichte RER wird in drei Studien berichtet [7,9, 13]. Unabhängig von der Altersgruppe, dem Geschlecht oder dem verwendeten Belastungsprotokoll überschritten alle untersuchten Personen zum Zeitpunkt der Ausbelastung die respiratorisch bestimmte anaerobe Schwelle deutlich ( $R E R \geq 1$ ).

\section{Der maximal erreichte Blutlaktatspiegel zur}

\section{Abschätzung der anaeroben Kapazität}

Die Angabe des unter der maximalen Belastung erreichten Laktats erfolgte in fünf Studien $[7,8,16,21,22]$. Saltin et al. [16] bestimmten den maximalen Laktatwert bei Männern zwischen 60 und 67 Jahren auf $10,7 \pm 3,0 \mathrm{mmol} / \mathrm{l}$. Der maximal erreichte Laktatwert bei den Frauen (Durchschnittsalter: 43 Jahre) lag bei 12,0 $\pm 0,3$ mmol/l, für Männer (Durchschnittsalter: 67 Jahre) bei 13,1 $\pm 0,4$ $\mathrm{mmol} / \mathrm{l}$ [21]. Drinkwater et al. [8] berichten über durchschnittliche Laktatkonzentrationen für Frauen zwischen 60 und 69 Jahren von $5,95 \pm 1,14 \mathrm{mmol} / \mathrm{l}$. Die von Sydney et al. [7] untersuchten durchschnittlich 63-jährigen Frauen wiesen eine Laktatkonzentration von $8,1 \pm 2,9 \mathrm{mmol} / \mathrm{l}$ und die durchschnittlich 64 -jährigen Männer von $10,3 \pm 3,6 \mathrm{mmol} / \mathrm{l}$ auf. In unserer Studie bestimmten wir den maximal erreichten Blutlaktatspiegel bei den Frauen mit $5,83 \pm 1,52 \mathrm{mmol} / \mathrm{l}$, bei den Männern mit $6,58 \pm 2,77 \mathrm{mmol} / \mathrm{l}$. Somit können die Daten von Drinkwater et al. [8] bei Frauen $(5,95 \pm 1,14 \mathrm{mmol} / \mathrm{l})$ und von Saltin et al. [16] bezüglich der 60bis 67-jährigen Männer auf einer Belastungsstufe von 147 Watt $(6,4 \pm 1,7 \mathrm{mmol} / \mathrm{l})$ bestätigt werden. Die maximal bei Männern beschriebenen Laktatkonzentrationen von über $10 \mathrm{mmol} / \mathrm{l}[7$, $16,21]$ konnten von uns nur in Einzelfällen gemessen werden und sprechen für eine begrenzte anaerobe Kapazität mit vermindertem Stehvermögen bei Schnelligkeit.

\section{Die alveolar-arterielle Gaspartialdruckdifferenz für} Sauerstoff $\left(\mathrm{AaDO}_{2}\right)$ zur Beurteilung des Gasaustausches Die $\mathrm{AaDO}_{2}$ stellt den simultan bestimmten Gradienten zwischen den endexpiratorischen Werten des Alveolarpartialdruckes für Sauerstoff und dem arteriellen Sauerstoffdruck in $\mathrm{mmHg}$ dar. Er beschreibt die tatsächliche Druckdifferenz, die der Sauerstoff von der Alveole in die pulmonalen Kapillaren überwinden muss. Die $\mathrm{AaDO}_{2}$ liegt im Allgemeinen zwischen 20 und $35 \mathrm{mmHg}$, bei über 50-Jährigen bis $35 \mathrm{mmHg}$ [22]. Hansen et al. [20] bestimmten die $\mathrm{AaDO}_{2}$ bei normalgewichtigen Probanden $(100-109 \%$ des Idealgewichtes) zu $17 \pm 10 \mathrm{mmHg}$. Pothoff et al. [5] berichten bei den 60- bis 69-jährigen Männern eine $\mathrm{AaDO}_{2}$ von $27 \pm 11 \mathrm{mmHg}$, bei den gleichaltrigen Frauen von $21 \pm 8 \mathrm{mmHg}$. In unserer Studie lag die $\mathrm{AaDO}_{2}$ in der Gruppe der 60- bis 69-jährigen Frauen bei $19 \pm 7$ $\mathrm{mmHg}$, bei den Männern bei $19 \pm 8 \mathrm{mmHg}$. Bei den Männern über 70 Jahre fällt die $\mathrm{AaDO}_{2}$ sogar auf $12 \pm 6 \mathrm{mmHg}$ ab, bei den Frauen hingegen steigt sie auf $21 \pm 7$ geringgradig an.

\section{Der Sauerstoffpartialdruck sowie der Kohlen- dioxidpartialdruck in Ruhe und unter Belastung zur Beurteilung der respiratorischen Funktion}

Der Sauerstoffpartialdruck in Ruhe liegt bei allen untersuchten Frauen im Mittel bei $81,4 \pm 8,7 \mathrm{mmHg}$ und bei den Männern bei $83,4 \pm 7,8 \mathrm{mmHg}$. Bei den Frauen fällt er unter Belastung geringgradig von der jüngeren zur älteren Gruppe von $82,4 \pm 9,8$ auf $79,3 \pm 6,3 \mathrm{mmHg}$ ab $(R=-0,1)$. Bei den Männern steigt er hingegen von $82,0 \pm 7,0$ auf $85,7 \pm 8,9 \mathrm{mmHg}$ nicht signifikant an $(r=0,24)$. Woitowitz et al. [23] beschrieben bei berufstätigen Männern und Frauen vor und gegen Ende abgestuft dosierter Arbeitsbelastung einen altersabhängigen Abfall des Sauerstoff- partialdruckes. Die Formeln zur Ermittlung der Sollwerte betragen für Frauen: $\mathrm{PaO}_{2}=-0,32 \times$ Alter $+98 \mathrm{mmHg}( \pm 10)$, für Männer $\mathrm{PaO}_{2}=-0,33 \times$ Alter $+100 \mathrm{mmHg}( \pm 10)$. Somit errechnet sich anhand dieser Formel für die Frauen mit einem Durchschnittsalter von 66 Jahren ein Sollwert von $76,88 \pm 10 \mathrm{mmHg}$. Bei den Männern mit einem Durchschnittsalter von 67 Jahren ergibt sich ein Sollwert von $77,89 \pm 10 \mathrm{mmHg}$. Ulmer et al. [24] berichten eine körpergewichtsbezogene (Broca-Index ${ }^{1}$ ) Sollwertformel für Frauen mit $\mathrm{PaO}_{2}=108,9-0,26 \times$ Alter $-0,073 \times$ BrocaIndex $\mathrm{mmHg}( \pm 14)$, für Männer $\mathrm{PaO}_{2}=109,4-0,26 \times$ Alter $-0,98 \times$ Broca-Index mmHg $( \pm 15)$. Bezogen auf unser Patientenkollektiv ergäbe sich hieraus für die Gruppe der Frauen ein Normwert von $90,95 \pm 14 \mathrm{mmHg}$, für die Männer von $88,59 \pm 15 \mathrm{mmHg}$. Der Sauerstoffpartialdruck unter maximaler Last lag in unserer Studie bei den Frauen bei $92,6 \pm 7,9 \mathrm{mmHg}$, bei den Männern bei $98,7 \pm 10,5 \mathrm{mmHg}$. Somit liegen die von uns bestimmten Sauerstoffpartialdrucke in den dargelegten Normbereichen. Die Altersabhängigkeit, dargestellt am Korrelationskoeffizienten ( $r$ ), war in unserem Kollektiv für Frauen $(r=-0,14)$ und für Männern $(r=$ $0,14)$ marginal.

Sämtliche Kohlendioxidpartialdrucke in Ruhe und unter Belastung lagen im Referenzbereich für Frauen von $32-43 \mathrm{mmHg}$ und für Männer von 35-46 mmHg [25].

\section{Fazit}

Die an jüngeren Kollektiven erhobenen spiroergometrisch ermittelten leistungsphysiologischen Parameter können nicht ohne weiteres auf Personen im Alter von über 60 Jahren übertragen werden. Bei der Feststellung von Leistungseinschränkungen in der sozialmedizinischen Begutachtung sollten altersentsprechende Referenzwerte verwendet werden. Die von uns bestimmten blutgasanalytisch erhobenen Parameter lagen in den bisher berichteten Normbereichen. Eine Altersabhängigkeit fand sich insbesondere für die Bruttoleistungsfähigkeit, die körpergewichtsbezogene Leistungsfähigkeit, die maximale Sauerstoffaufnahme und die körpergewichtsbezogene Sauerstoffaufnahme bei Männern.

\section{Interessenkonflikt}

$\nabla$

Die Autoren geben an, dass kein Interessenkonflikt besteht.

\section{Literatur}

1 Preisser AM, Ochmann U. Die Spiroergometrie in der arbeitsmedizinischen Eignungsuntersuchung und Begutachtung. Pneumologie 2011; 65: $662-670$

2 Kroidl RF, Nowak D, Seysen U. Bewertung und Begutachtung in der Pneumologie. Stuttgart: Thieme; 2000

3 Mader $H$, Liesen $H$, Heck $H$ et al. Zur Beurteilung der sportspezifischen Ausdauerleistungsfähigkeit im Labor. Sportarzt und Sportmed 1976; 27: 109

4 Blackie SP, Fairbarn MS, McElvaney GN et al. Prediction of maximal oxygen uptake and power during cycle ergometry in subjects older than 55 years of age. Am Rev Respir Dis 1989; 139: 1424-1429

5 Pothoff G, Winter U, Wassermann K et al. Ergospirometrische Normalkollektivuntersuchungen für Unsteady-state-Stufentestprogramme. Z Kardiol 1994; 83: 116-123

\footnotetext{
${ }^{1}$ Körpergröße in cm - $100=$ Normalgewicht
} 
6 Ong $K C$, Loo $C M$, Ong $Y Y$ et al. Predictive values for cardiopulmonary exercise testing in sedentary Chinese adults. Respirology 2002; 7: $225-231$

7 Sidney KH, Shephard RJ. Maximum and submaximum exercise tests in men and women in the seventh, eighth and ninth decades of life.J Appl Physiol: Respirat Environ Exercise Physiol 1977; 43: 280-287

8 Drinkwater BL, Horvath SM, Wells CL. Aerobic power of females, ages 10 to 68. J Gerontology 1975; 30: 385-394

9 Jones NL, Makrides L, Hitchcock C et al. Normal standards for an incremental progressive cycle ergometer test. Am Rev Respir Dis 1985; 131: $700-708$

10 Fairbarn MS, Blackie SP, McElvaney NG et al. Prediction of heart rate and oxygen uptake during incremental and maximal exercise in healthy adults. Chest 1994; 105: 1365-1369

11 Neder JA, Nery LE, Castelo A et al. Prediction of metabolic and cardiopulmonary responses to maximum cycle ergometry: a randomised study. Eur Respir J 1999; 14: 1304-1313

12 Posner JD, Gorman KM, Klein HS et al. Ventilatory threshold: measurement and variation with age. J Appl Physiol 1987; 63: 1519-1525

13 Herdy $A H$, Uhlendorf $D$. Reference values for cardiopulmonary exercise testing for sedentary and active men and women. ARER Bras Cardiol 2011; 96: 54-59

14 Koch B, Schäper C, Ittermann $T$ et al. Reference values for cardiopulmonary exercise testing in healthy volunteers: the SHIP study. Eur Respir J 2009; 33: 389- 397

15 American Heart Association. Exercise Testing and Training of Apparently Healthy Individuals. 2nd: ed. Dallas, Texas: 1972: 13
16 Saltin B, Grimby G. Physiological analysis of middle-aged and old former athletes: comparison with still active athletes of the same ages. Circulation 1968; 38: 1104-1115

17 McDonough JR, Kusumi F, Bruce RA. Variations in maximal oxygen intake with physical activity in middle-aged men. Circulation 1970; 41 : $743-752$

18 Funk $M$, Arhelger R, Brandt-Younis $S$ et al. Laktatbestimmungen zur arbeits- und sozialmedizinischen Abschätzung des körperlichen Restleistungsvermögens bei Patienten mit Atemwegs- und Lungenerkrankungen. Arbeitsmed Sozialmed Umweltmed 2011; 46: 656-661

19 Takeshima N, Kobayashi F, Watanabe T et al. Cardiorespiratory responses to cycling exercise in trained and untrained elderly: with special reference to the lactate threshold. Appl Human Sci 1996; 15: 267-273

20 Hansen JE, Sue DY, Wassermann K. Predicted values for clinical exercise testing. Am Rev Respir Dis 1984; 129: 49-55

21 Åstrand I, Astrand P-O, Hallbäck I et al. Reduction in maximal oxygen uptake with age. J. Appl Physiol 1973; 35: 649-654

22 Löllgen H, Erdmann E, Gitt A Hrsg. Ergometrie, Belastungsuntersuchungen in Klinik und Praxis. 3. Aufl. Heidelberg: Springer; 2010

23 Woitowitz H-J, Schäcke G, Woitowitz RH. Arterielle Blutgase berufstätiger Männer und Frauen vor und gegen Ende abgestuft dosierter Arbeitsbelastung. Int Arch Gewerbepathol Gewerbehyg 1969; 25: 124 139

24 Ulmer WT, Reichel G. Untersuchungen über die Altersabhängigkeit der alveolären und arteriellen Sauerstoff- und Kohlensäuredrücke. Klin Wschr 1983; 41: 1-6

25 Thomas L. Labor und Diagnose. 6. Aufl. Frankfurt: Th-Books; 2005 